\title{
Teaching Electroencephalography: Persistent Altered Sensorium with Ominous Appearing Electroencephalographic Activity
}

\author{
Delon D’Souza' Gosala R. K. Sarma ${ }^{1}$ Elizabeth V.T.
}

Address for correspondence Gosala R. K. Sarma, DM (Neurology), Department of Neurology, St. John's Medical College Hospital, Sarjapura road, Bengaluru, Karnataka 560034, India (e-mail: grk_sarma@yahoo.com).

\author{
Abstract \\ Keywords \\ - electroencephalogram \\ - lateralized periodic \\ discharges \\ - nonconvulsive status \\ epilepticus \\ - Salzburg's criteria
}

A 51-year-old man presented with persistent altered sensorium following a seizure. His magnetic resonance imaging (MRI) showed features of focal encephalitis involving the left temporal, parietal, and occipital regions. His electroencephalogram (EEG) showed ongoing epileptiform discharges over the left hemisphere. This article discusses dilemmas in the diagnosis of nonconvulsive status epilepticus in such a case scenario.
A 51-year-old man was admitted with repeated right facial and upper limb clonic seizures with persistent drowsiness of 1-day duration. Convulsions were controlled with antiepileptic drugs, but the patient continued to be in altered sensorium. He did not have any subtle clinical seizure activity. Magnetic resonance imaging (MRI) of the brain showed left parieto-occipital gyriform signal changes with contrast enhancement suggestive of focal encephalitis (-Fig. 1). His electroencephalogram (EEG) tracing is shown in - Fig. 2.

\section{Questions}

1. Describe the EEG findings and the possible EEG differential diagnosis.

2. Which EEG clinical criteria could help differentiate the two possible conditions?

3. Applying these criteria, what is the most likely diagnosis in this patient?

\section{Answers}

1. EEG shows periodic, left hemispheric, high-amplitude epileptiform discharges at 1 - to $1.5-\mathrm{Hz}$ frequency. They are fairly monomorphic with a spike-wave morphology, best seen over the left parietal, temporal, and occipital regions. The two important differential diagnoses are

a. Lateralized periodic discharges

b. Possible nonconvulsive status epilepticus (NCSE)

2. The following modified Salzburg's criteria for NCSE are laid down to help in such situations ${ }^{1}$ :

\section{Clinical Data}

- Clinical deterioration within minutes to hours.

- No significant improvement in the patient's condition in the past few minutes or hours.

- Brain imaging findings cannot account for EEG findings.

- No metabolic derangement or toxic etiology to account for EEG findings.

Electroencephalogram Data: EEG changes have to be continuously present for $\geq 10$ seconds.

A. In patients without known epileptic encephalopathy (one of the criteria $1-3$ should be fulfilled).

1. Epileptiform discharges (EDs) $>2.5 \mathrm{~Hz}$

2. Typical ictal spatiotemporal evolution of EDs or rhythmic activity $(>0.5 \mathrm{~Hz})$ received

October 8, 2018

accepted after revision

October 10, 2018

published online

January 07, 2019
DOI https://doi.org/

$10.1055 / \mathrm{s}-0038-1676560$

ISSN 2213-6320.
Copyright (C2018 Indian Epilepsy Society
License terms

() (1) $\ominus \circledast$ 


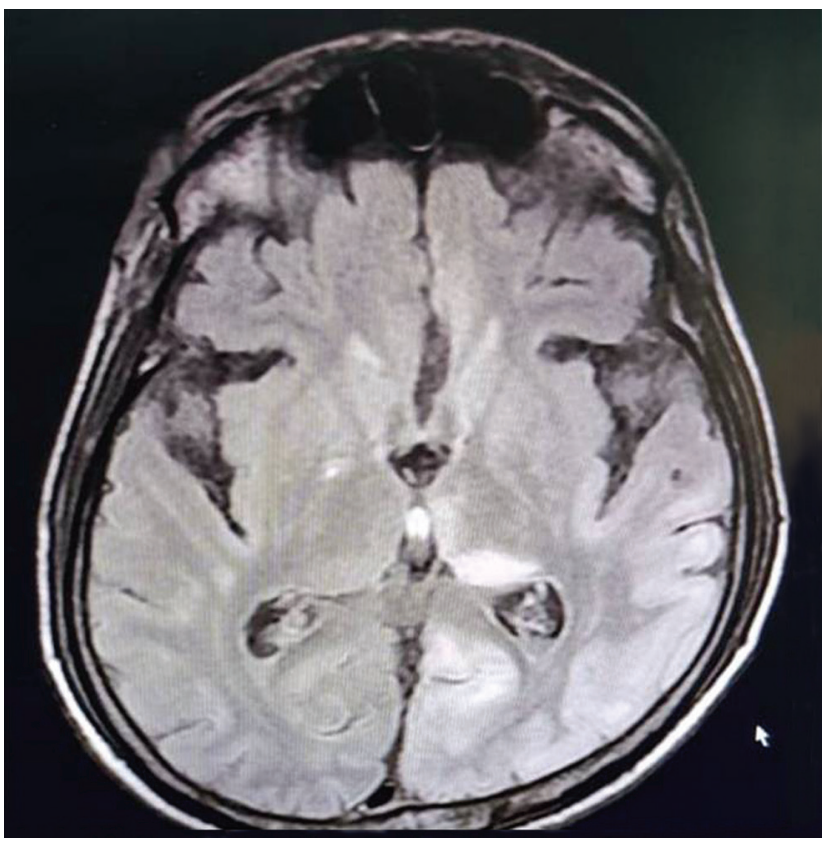

Fig 1. Axial FLAIR MRI showing left thalamic and temporo-parietooccipital gyriform hyperintensity. These areas showed enhancement with Gadolinium (not shown in the figure). FLAIR MRI, fluid-attenuated inversion recovery magnetic resonance imaging.

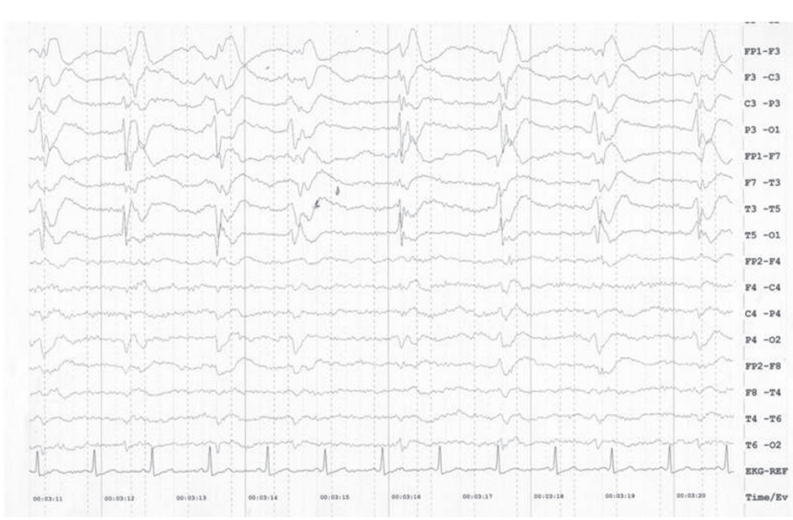

Fig 2. Electroencephalogram tracing.

3. Subtle ictal clinical phenomena with EDs or rhythmic activity $(>0.5 \mathrm{~Hz})$

4. If criteria 1 to 3 are not fulfilled but one of the following patterns is present, apply appropriate antiepileptic drug (AED) and document response:

(4a) EDs $\leq 2.5 \mathrm{~Hz}$ with fluctuation or

(4b) Rhythmic activity $(>0.5 \mathrm{~Hz})$ with fluctuation or

(4c) Rhythmic activity $(>0.5 \mathrm{~Hz})$ without fluctuation

Clinical improvement is defined as better performance in one of five domains: (1) "say your surname"; (2) "repeat 1,2, 3"; (3) "raise your arms"; (4) the patient opens eyes to 1-3; and (4) the patient looks at the examiner in response to $1-3$.
If there is no response, repeat procedure after strong tactile stimuli on both sides of the body.

EEG improvement is defined as reduction to "occasional," i.e., 1 to $9 \%$ of epoch.

Document response:

- No EEG improvement and no clinical improvement

- Only EEG improvement but no clinical improvement

- Only clinical improvement but no EEG improvement

- EEG and clinical improvements

This response has no bearing on diagnosis of NCSE, which depends on criteria 1 to 3 only.

B. In patients with known epileptic encephalopathy.

In addition to the criteria above $(A)$, these patients have to fulfill one of the following:

a. Increase in prominence or frequency of EEG discharges when compared to baseline with observable change in clinical state

b. Improvement of clinical and EEG features with intravenous AEDs

Spatiotemporal evolution includes

- Incremental onset (increase in amplitude and frequency)

- Evolution in pattern: change in frequency $>1 \mathrm{~Hz}$ or change in location

- Decremental termination (reduced amplitude and frequency)

3. In this patient, the epileptiform discharges occurred at less than $2.5 \mathrm{~Hz}$ without fluctuation or spatiotemporal evolution or any subtle seizure activity. Thus, the modified Salzburg's criteria for NCSE were not fulfilled. Four mg of intravenous lorazepam was given without any change in clinical condition or EEG appearance. The EEG pattern thus favors lateralized periodic discharges (LPDs) over NCSE.

Periodic discharges are repetitive discharges that occur at fairly constant intervals. These discharges may be generalized, lateralized multifocal, or bilateral independent periodic. ${ }^{2}$ They are considered to be in the ictal-interictal continuum. LPDs can occur due to any underlying destructive cortical lesion, such as infarct, viral encephalitis, Creutzfeldt-Jakob disease (CJD), brain tumors, or hematomas.

\section{Conflict of Interest}

None declared.

\section{References}

1 Leitinger M, Beniczky S, Rohracher A, et al. Salzburg consensus criteria for non-convulsive status epilepticus-approach to clinical application. Epilepsy Behav 2015;49:158-163

2 Lin L, Drislane FW. Lateralized periodic discharges: a literature review. J Clin Neurophysiol 2018;35(3):189-198 